\title{
A SEMI-MARKOV MODEL FOR THE DURATION OF STAY IN A NON- HOMOGENOUS ACADEMIC MANPOWER SYSTEM
}

A. U. UDOM

(Received 7 January 2009; Revision Accepted 4 March 2009)

\section{ABSTRACT}

The semi-Markov approach to a non-homogenous manpower system is considered. The mean duration of stay in a grade and the total duration of stay in the system are obtained. A renewal type equation is developed and used in deriving the limiting distribution of the semi Markov process. Empirical estimators of the semi-Markov parameters which are uniformly consistent and asymptotically normally distributed are used to obtain the average length of stay for the academic manpower of the University of Nigeria.

KEYWORDS: Duration of stay, Manpower, Non homogeneous Semi-Markov, Uniformly consistent,

\section{$1 \quad$ INTRODUCTION}

The problem of disaggregating heterogeneity in manpower systems has been discussed by a number of authors. This disaggregation of a manpower system normally correspond to some classification of the manpower system into homogenous subgroups as for example, in the institution with grades of staff discussed by Young and Almond (1961), the classification of manpower system by age as in Uche and Udom (2006) and the classification of a system by degrees of commitment to the system, for example Bartholomew (1971) and McClean (1976)

Transition models based on the Markov theory and renewal theory have been used in the description of these heterogeneous populations; see for example Bartholomew (1982). Most of these models are transition models and are concerned with the effect of constant transition probabilities on changing class structure. These models enable us to see how the aggregate behaviour of the system depends on the propensity to move its members. These models however contain some fundamental weaknesses. For instance, the simple Markov model assumes that transition from one grade to another or leaving occurs at a constant rate, thus the completed length of service (CLS) in grade i prior to leaving has a degenerate geometric distribution. This assumption inherent in the simple Markov model does not apply to many situations. A generalization of these models is the semi-Markov model in which we have a combination of transition probabilities of moving between grades with the conditional distribution of length of stay in a grade before transition to the next.

McClean (1978), formulated a continuous time stochastic model for a multi-grade population by extending the continuous time Markov model of a multi-grade system to a semiMarkov formulation which incorporated the internal transitions and the leaving process in the system. Jain (1989), developed a methodology that can be used to study the length of stay distribution of cancer of cervix patients in various states of the disease. A similar use of this methodology can be found in manpower modelling. Uche (1990) examined the effect of heterogeneity on Markovian analysis and stressed the need to disaggregate population in order to ensure homogeneity or a reasonable level of homogeneity. Ugwuowo and McClean (2000), reviewed methods of incorporating population heterogeneity into manpower modeling and emphasized the analysis of differentials in a manpower system since they are sources of aggregation error in stochastic models. Two strategies were stressed, the use of observable sources of heterogeneity as they affect wastage and latent sources of heterogeneity which cannot be identified precisely but are known to affect the key parameters of most models.

In this paper, we develop a semi-Markov model to be used in describing a nonhomogenous manpower system, obtain the limiting behavior of the semi-Markov process and use 
the model to obtain the average duration of stay in the various academic grades, and total duration of stay in the system for the University of Nigeria Academic Manpower system.

\section{THE SEMI-MARKOV MODEL}

Consider a $\mathrm{k}$ graded organisation where transition from one grade to another is governed by a semi-Markov process. Let the transient grades be $S_{1}, S_{2}, \ldots, S_{k}$; the probability of moving from grade $i$ to $j$ be $P_{i j}(i, j=1,2, \ldots, k)$ where $P=P_{i j}$ is a stochastic matrix. Let $t_{i j}$ represent the length of time an employee stays in grade $\mathrm{i}$ before transition to $\mathrm{j}$, given that the transition eventually occurs. Thus $t_{i j}$ is a random variable whose density function $f_{i j}(t)$ is completely determined by transition probabilities and holding time distributions of the semi-Markov process. The corresponding distribution and survivor functions are $F_{i j}(t)$ and $G_{i j}(t)=1-F_{i j}(t)$.

Let $r_{i j}(t) d t(i \neq j)$ be the instantaneous rate of transition from grade $j$ within an infinitesimal interval of time $\mathrm{dt}$, conditioned on movement to $\mathrm{j}$ from $\mathrm{i}$. Assuming independent risk, we define

$r_{i}(t)=\sum_{j \neq i} r_{i j}(t)$ to be the instantaneous rate of departure from grade $\mathrm{i}$ at time $\mathrm{t}$ given stay in $\mathrm{i}$ until $\mathrm{t}$.

Notice that $r_{i j}(t)$ is a decreasing function of $t$. The corresponding density function of the duration of stay in grade $i$ is $f_{i}(t)$ with distribution function $F_{i}(t)$ and

survivor function

$$
\mathrm{G}_{\mathrm{i}}(\mathrm{t})=1-\mathrm{F}_{\mathrm{i}}(\mathrm{t}) \quad=\int_{t}^{\infty} f_{i}(x) d x
$$

Thus,

$$
\begin{array}{r}
r_{i}(t) \partial(t)=\frac{f_{i}(t) d t}{G_{i}(t)} \\
=\frac{P[\text { leaves grade } i \text { within } \partial t]}{P[\text { still in grade } i \text { at } t]} \\
\text { So } r_{i}(t)=\partial / \partial t\left[-\log G_{i}(t)\right] \\
\text { and } G_{i}(t)=\exp \left\{-\int_{0}^{t} r_{i}(x) d x\right\} \\
f_{i}(t)=r_{i}(t) \exp -\int_{0}^{t} r_{i}(x) d x
\end{array}
$$

Thus $r_{i}(t), G_{i}(t)$ and $f_{i}(t)$ are related in the following from

$$
\mathrm{r}_{\mathrm{i}}(t)=\frac{f_{i}(t)}{G_{i}(t)}
$$

The probability of eventually making transition from grade $i$ to grade $j$ is therefore given by

$$
\begin{aligned}
P_{i j}=\int_{0}^{\infty} r_{i j}(t) G(t) d t ;(i \neq j) & \\
& \Rightarrow P_{i j}=\int_{0}^{\infty} r_{i j}(t) \exp \left\{-\int_{0}^{t} r_{i}(x) d x\right\} \partial t
\end{aligned}
$$

The probability density function of length of stay in grade $\mathrm{i}$ before moving to grade $\mathrm{j}$ condition on such a move eventually taking place is

$$
f_{i j}(t)=\frac{r_{i j}(t) \exp \left\{-\int_{0}^{t} r_{i}(x) d x\right\}}{P_{i j}} .
$$


Since $f_{i j}(t) d t=P$ [person in i moves to $j$ in $(t, t+d t) \mid$ next move is to $\left.j\right]$

$$
=\frac{r_{i j}(t) G_{i}(t)}{P_{i j}}
$$

This model can be used to predict the future stocks in each grade at a future time point by noting that $P_{\mathrm{ij}}(t)$ is the probability, that an individual is in grade $\mathrm{j}$ at time $t$ conditioned on his being in grade $\mathrm{i}$ at time $\mathrm{t}=0$.

$$
\begin{aligned}
P_{i j}(t)= & \sum_{r=1}^{k} \int_{0}^{t} P_{i j} f_{i r}(x) P_{i r}(t-x) d x+U_{i j} \sum_{r \neq i} P_{i r} G_{i r}(t) \\
= & \sum_{r=1}^{k} \int_{0}^{t} g(t) P(t-x) d x+U_{i j} \sum_{r \neq i} H(t)
\end{aligned}
$$

where $U_{i j}=\left\{\begin{array}{cc}1 & \text { if } i=j \\ 0 & \text { otherwise }\end{array}\right.$

By obtaining a renewal type equation for $\mathrm{P}_{\mathrm{ij}}(\mathrm{t})$ and solving using Laplace transform we have that $P^{*}(s)=\left[I-g^{*}(s)\right]^{-1} H^{*}(s)$

where ${ }^{*}$ denotes the Laplace transform.

Let $\hat{n}_{j}(t)$ be the expected number in grade $\mathrm{j}$ at time $\mathrm{t}$. Then

$\hat{n}_{j}(t)=\sum_{i=1}^{k}\left(n_{i}(0) P_{i j}+\int_{0}^{t} \bar{N}_{R} P_{o i} P_{i j}(t-x) d x\right)$

where $\bar{N}_{R}$ is the expected number of recruits in $(x, x+d x)$ and $\mathrm{P}_{\mathrm{oi}}$ is the recruitment probability into grade $i$.

\section{AVERAGE LENGTH OF STAY IN GRADE J}

Let $\phi_{\mathrm{ij}}(\mathrm{t})$ be the probability that an employee will go into grade $\mathrm{j}$ within $\mathrm{t}$ time units after entering grade $i$. Then the expected duration of stay in grade $\mathrm{j}$ is given by

$$
\begin{aligned}
\bar{t}_{i j}=E\left(t_{i j}\right) & =E\left(\sum_{t=1}^{\infty} u_{i j}(t)\right) \\
& =\sum_{t=0}^{\infty} E\left[u_{i j}(t)\right]
\end{aligned}
$$

where $u_{i j}$ is an indicator variable defined by

$u_{i j}= \begin{cases}1 & \text { when an employee is in grade } j \text { at time } t \text { given that he was in grade } i \text { at time zero } \\ 0 & \text { otherwise }\end{cases}$

The probability that $u_{i j}(t)=1$ is $\phi_{i j}(t)$ and the probability that $u_{i j}(t)=0$ is $1-\phi_{i j}(t)$.

Thus

$$
E\left[U_{i j}(t)\right]=\phi_{i j}(t)
$$

Hence

$$
E\left(t_{i j}\right)=\sum_{t=0}^{\infty} \phi_{i j}(t)=(I-P)^{-1} M
$$

where $\mathrm{M}$ is a diagonal matrix of mean waiting time in grade $\mathrm{j}$. 
To obtain the limiting distribution of a semi - Markov process, it is not enough to obtain the limit of the $P_{i j}(t)$. Here, we develop a renewal type equation for the process by considering the problem of finding the limit, as $t \rightarrow \infty$, of the probability of being in grade $\mathrm{j}$ at time $\mathrm{t}$, of making the next transition before $\mathrm{t}+\mathrm{x}$ and of this transition being into grade $\mathrm{k}$.

Let $L(t)=P\{Z(t)=j, Z(t+1)=k, Y(t) \leq x \mid Z(0)=j\}$ be the probability when the initial grade is $\mathrm{j}$, where $Y(t)$ is the time starting from $t$ until the next transition. Conditioning on $T$ the time of the first transition to grade $\mathrm{j}$, we have the following renewal type equation

$L(t)=\int_{0}^{t} L(t-r) d G_{j j}(r)+h(t)$

where $h(t)=\int_{0}^{\infty} P\left\{t<X_{1} \leq t+x, Z(t+1)=k \mid Z(0)=j, T=r\right\} d G_{j j}$

Solving for $L(t)$ we have,

$L(t)=h(t)+\int_{0}^{t} h(t-x) d m(x)$

where $m(x)=\sum_{n=1}^{\infty}\left(G_{j j}\right)_{n}(x)$

By the key renewal theorem we have that

$$
\begin{aligned}
\operatorname{Lim}_{t \rightarrow \infty} L(t) & =\int_{0}^{\infty} \frac{h(t)}{\mu_{j j}} \\
& =\frac{1}{\mu_{j j}} \int_{0}^{\infty} P\left[t<X_{1} \leq t+x, Z(t+1)=k \mid Z(0)=j\right] d t \\
& =\frac{1}{\mu_{j j}} \int_{0}^{\infty} P_{j k}\left[F_{j k}(t+x)-F_{j k}(t)\right] d t \\
& =\frac{P_{j k}}{\mu_{j j}} \int_{0}^{\infty}\left[1-F_{j k}(t)-\left\{1-F_{j k}(t+x)\right\}\right] d t
\end{aligned}
$$

Thus $\operatorname{Lim}_{t \rightarrow \infty} L(t)=\frac{P_{j k}}{\mu_{j j}} \int_{0}^{x}\left[1-F_{j k}(t)\right] d t$

\section{APPLICATION}

In this section we apply the semi-Markov model described in the last section to the academic manpower data of the University of Nigeria Nsukka obtained from the Personnel Services Department of the University. The data consist of manpower flows between grades and duration of stay in a grade before transition from 1998 to 2004.

We used the empirical estimators of the semi-Markov Kernel defined by 


$$
\hat{Q}_{i j}(t)=\frac{1}{N_{i}(t)} \sum_{i=1}^{N(t)} 1_{i, j}
$$

from our definition

$$
\hat{Q}_{i j}(t)=\hat{P}_{i j}(t) G_{i j}(t)
$$

$$
\begin{gathered}
\text { where } \hat{P}_{i j}(t)=\frac{N_{i j}(t)}{N_{i}(t)} \\
N_{i}(t)=\sum_{i \neq j} N_{i j}(t) \\
\hat{G}_{i j}(t)=\frac{1}{N_{i j}(t)} \sum_{i=1}^{N_{i j}(t)} 1_{i, j}
\end{gathered}
$$

These estimators of the semi-Markov parameters have been shown by Limnios and Oprison (2001), to be uniformly strongly consistent and converge asymptotically to normal random variables in the sense that

$$
\underset{t \rightarrow \infty}{\operatorname{Limmaxsup}}\left|\hat{Q}_{i j \in[0, t)}(x, t)-Q_{i j}(x)\right|=0 \text { almostsurely. }
$$

The results of our estimations are as summarized below:

The matrix of mean duration of stay in the ith grade

$$
M=\left[\begin{array}{ccccccc}
5.62 & 0.00 & 0.00 & 0.00 & 0.00 & 0.00 & 0.00 \\
0.00 & 5.84 & 0.00 & 0.00 & 0.00 & 0.00 & 0.00 \\
0.00 & 0.00 & 6.34 & 0.00 & 0.00 & 0.00 & 0.00 \\
0.00 & 0.00 & 0.00 & 7.86 & 0.00 & 0.00 & 0.00 \\
0.00 & 0.00 & 0.00 & 0.00 & 11.21 & 0.00 & 0.00 \\
0.00 & 0.00 & 0.00 & 0.00 & 0.00 & 10.97 & 0.00 \\
0.00 & 0.00 & 0.00 & 0.00 & 0.00 & 0.00 & 12.48
\end{array}\right]
$$

Table showing the average and total duration of stay in the system

\begin{tabular}{|l|l|l|}
\hline Grades & $\begin{array}{l}\text { Average duration of stay in the ith } \\
\text { grade }\end{array}$ & $\begin{array}{l}\text { Total duration of stay in the } \\
\text { system }\end{array}$ \\
\hline Graduate Assistant & 5.62 & 28.56 \\
\hline Assistant Lecturer & 5.84 & 25.43 \\
\hline Lecturer II & 6.34 & 19.28 \\
\hline Lecturer I & 7.86 & 18.35 \\
\hline Senior Lecturer & 11.21 & 15.69 \\
\hline Associate Professor & 10.97 & 14.29 \\
\hline Professor & 12.48 & 12.45 \\
\hline
\end{tabular}

\section{CONCLUSION}

We have described a semi-Markov model suitable for a non-homogenous manpower system and obtained an estimator for the average duration of stay in a grade before transition given that this transition eventually occurs, as well as the limiting distribution of the process. The empirical estimates of the duration of stay for the academic manpower data of University of Nigeria, show that the average duration of stay in the different academic grades increases hierarchically. However for the total duration of stay in the system, the reverse is the case. 


\section{REFERENCES}

Bartholomew, D.J., 1971. The statistical approach to manpower planning, The Statistician. 220: 3 26.

Bartholomew, D. J., 1982. Stochastic models for social processes. $3^{\text {rd }}$ ed. John Wiley and Sons Chichester.

Jain, R. K., 1989. A semi-Markov model for the average length of stay in transient states and its application. Computer and Biomedical research 22: 209-214.

Limnios, N. and Oprison, G., 2001. Semi-Markov processes and reliability. Birkhauser Boston.

McClean, S. I., 1976, The two stage model of personnel behaviour J. Roy Stat Soc. A 139: 205 217.

McClean, S. J., 1978. Continuous-time stochastic models for a multigrade population. J. Appl. Prob. 15: 26-37.

Uche, P. I., 1990. Non- homogeneity and transition probabilities of a Markov chain. Int. J. Math. Edu. Sci Tech 21, (2): 295 - 301

Uche, P. I. and Udom, A. U., 2006. Age dependent Markov model of manpower system. Global Journal of Mathematical Sciences 5, (2): 147 - 152 .

Ugwuowo F. I. and McClean, S., 2000. Modelling heterogeneity in a manpower system: A review. Applied Stochastic Models in Business and Industry, 16: $99-110$

Young, A. and Almond, G., 1961. Predicting distribution of staff . Comp. J. 3: $246-250$ 